\title{
Perilesional edema in brain metastases as predictive factor of response to systemic therapy in non-small cell lung cancer patients: a preliminary study
}

\author{
Montse Alemany ${ }^{1}$, Marta Domènech ${ }^{2}$, Andreas A. Argyriou ${ }^{3}$, Noelia Vilariño ${ }^{1,2}$, Carles Majós ${ }^{1,4}$, \\ Pablo Naval-Baudin ${ }^{4}$, Anna Lucas ${ }^{1}$, Ramón Palmero ${ }^{2}$, Marta Simó $^{1}$, Ernest Nadal ${ }^{2}$, Jordi Bruna $^{1}$ \\ ${ }^{1}$ Neuro-Oncology Unit, Hospital Universitari de Bellvitge-ICO L'Hospitalet (IDIBELL), Barcelona, Spain; ${ }^{2}$ Thoracic Oncology Unit, Catalan \\ Institute of Oncology, L'Hospitalet, Barcelona, Spain; ${ }^{3}$ Department of Neurology, Saint Andrew's State General Hospital of Patras, Patras, Greece; \\ ${ }^{4}$ Radiology Department ICS-IDI, Hospital Universitari Bellvitge, L'Hospitalet, Barcelona, Spain \\ Contributions: (I) Conception and design: M Alemany, M Domènech, E Nadal, J Bruna; (II) Administrative support: M Alemany, M Domènech; \\ (III) Provision of study materials or patients: C Majós, P Naval-Baudin, N Vilariño, R Palmero, M Simó; (IV) Collection and assembly of data: M \\ Alemany, M Domènech, E Nadal, J Bruna; (V) Data analysis and interpretation: M Alemany, M Domènech, AA Argyriou, E Nadal, J Bruna; (VI) \\ Manuscript writing: All authors; (VII) Final approval of manuscript: All authors. \\ Correspondence to: Jordi Bruna, MD, PhD. Neuro-Oncology Unit, Hospital Universitari de Bellvitge-ICO L'Hospitalet (IDIBELL), Barcelona, Spain. \\ Email: 35078jbe@comb.cat.
}

Background: The significance of upfront systemic therapies as an alternative to whole brain radiotherapy (WBRT) for multiple brain metastases (BM) is debatable. Our purpose is to investigate if peritumoral edema could predict the intracranial response to systemic chemotherapy (chemo) in patients with advanced nonsquamous non-small cell lung cancer (non-SQ-NSCLC) and synchronous multiple BM.

Methods: In this observational cohort study, we evaluated the outcome of 28 patients with multiple BM $(\geq 3)$ treated with chemo based on cisplatin/carboplatin plus pemetrexed (chemo, group A, n=17) or WBRT plus subsequent chemo (group $\mathrm{B}, \mathrm{n}=11$ ). The intracranial response, assessed by the response assessment neuro-oncology (RANO) BM criteria, was correlated with the degree of BM-associated edema estimated by the maximum diameter ratio among fluid attenuated inversion recovery (FLAIR) and gadolinium-enhanced T1WI (T1Gd) per each BM at the baseline brain magnetic resonance imaging (MRI).

Results: No differences were observed in baseline characteristics between both groups, except for the number of patients under steroid treatment that was clearly superior in group B $(\mathrm{P}=0.007)$. Median OS was similar between groups. Regarding FLAIR/T1Gd ratio (F/Gd), patients treated with chemo alone exhibited significantly higher values $(\mathrm{P}=0.001)$ in those who developed intracranial progression disease (PD) $(2.80 \pm 0.32 \mathrm{~mm})$, compared with those who achieved partial response $(\mathrm{PR})(1.30 \pm 0.11 \mathrm{~mm})$ or stable disease (SD) $(1.35 \pm 0.09 \mathrm{~mm})$. In patients treated with WBRT, F/Gd ratio was not predictive of response.

Conclusions: Peritumoral edema estimated by F/Gd ratio appears a promising predictive tool to identify oligosymptomatic patients with multiple BM in whom WBRT can be postponed.

Keywords: Brain metastases (BM); systemic chemotherapy; magnetic resonance imaging (MRI); non-small cell lung cancer (NSCLC); edema

Submitted Sep 18, 2020. Accepted for publication Jan 15, 2021.

doi: 10.21037/atm-20-6497

View this article at: http://dx.doi.org/10.21037/atm-20-6497 


\section{Introduction}

Brain metastases (BM) are the most frequent type of brain tumor in adults, accounting for $10-30 \%$ of all adult solid tumors (1-3). Approximately $50 \%$ of all BM account for lung cancer (4). Regarding non-small cell lung cancer (NSCLC), the incidence of BM represents 10-20\% among newly diagnosed patients and reaches 30-40\% in subsequent diagnoses after the primary tumor $(5,6)$. BM survival prognosis differs among tumor histology subtypes, representing less than 2 years in cases of nononcogene addicted NSCLC. Fortunately, this dismal prognosis is changing as a result of an early BM diagnosis in asymptomatic or oligosymptomatic stages (7).

Historically, patients with multiple BM have been excluded from most clinical trials and their therapeutic approaches have usually been palliative; with whole brain radiation therapy (WBRT) being the standard of care (8). Nevertheless, the evidence on the efficacy and benefit of WBRT is poor. The first conducted studies were series of cases from the 50s and 60s (9). From this date onwards, only two randomized, phase 3 clinical trials have addressed this question; one conducted in the pre-computed tomography scan era (10), and the most recent, despite improving local control, without showing any significant impact on patients' quality of life and survival rates (11). In addition, as most BM patients were supposed to have a poor prognosis, WBRT toxicity studies, such as neurocognitive dysfunction, have been limited (12). However, the improvement in overall survival (OS) combined with recent results on early cognitive impairment have increased the relevance of neurocognition monitoring and maintenance (13). In this setting, systemic therapies, such as conventional chemotherapy (chemo) or less controversial immunotherapies with immune checkpoint inhibitors (ICIs) (14), have become an alternative approach to WBRT in patients with multiple BM.

In the era of pre-molecular testing, clinical trials using chemo had already been conducted, including patients with multiple asymptomatic to oligosymptomatic BM from NSCLC (single arm-phase II and randomized phase III studies). The findings of these studies revealed a clinically meaningful intracranial activity with brain response rates of $30-40 \%$ in platinum-pemetrexed based chemo regimens (15-17). In addition, development of new targeted therapies for oncogene-addicted NSCLC has also contributed to increase the intracranial response rates (18-20). Similar effects have been reported by using systemic therapies with other tumor types, as HER2-positive breast cancer (21) and $B R A F \mathrm{~V} 600 \mathrm{E}$-mutated melanoma patients (22-24). On the other hand, immunotherapy can be an alternative frontline treatment for patients without driver oncogenes as long as the tumour expresses PD-L1 equal or superior to $50 \%$ (25-27). Additionally, immunotherapy in combination with chemo has also been approved as a frontline treatment regardless of PD-L1 expression $(14,28,29)$. Unfortunately, apart from a two-phase II trials $(30,31)$ and a pooled analysis of KEYNOTE-189 (32) none of the studies have been specifically designed to evaluate the intracranial response in patients with advanced NSCLC and multiple BM.

From a biological perspective, the blood-brain barrier (BBB) is structurally disrupted and functionally impaired with metastatic tumor growth, among others, by matrix tumor cells metalloproteinases (5). However, the potential increase in the permeability may be counterbalanced by the edema of surrounding tumor cells. The interstitial fluid pressure generated by this peritumoral edema might hinders the adequate drug diffusion to central nervous system (CNS). Towards this view, the interplay between these two major forces would be a determinant on the final intracranial response.

The aim of this current exploratory study was to investigate if peritumoral edema could have a predictive role on intracranial response to systemic chemo in patients with newly diagnosed non-squamous non-small cell lung cancer (non-SQ-NSCLC) and synchronous multiple BM. We present the following article in accordance with the STROBE reporting checklist (available at http://dx.doi. org/10.21037/atm-20-6497).

\section{Methods}

\section{Cobort study}

The study was conducted in accordance with the Declaration of Helsinki (as revised in 2013). The study was approved by institutional research committee (PR005/20) and individual consent for this retrospective analysis was waived.

Patients were recruited retrospectively from the Thoracic Oncology Unit, Catalan Institute of Oncology, L'Hospitalet de Llobregat between 2013 and 2016. Demographic and clinical characteristics, baseline and longitudinal (every 3 months) MRI images were collected together with OS data. All patients, comprising our study sample were followed for at least 1 year. 


\section{Participants}

Eligible for inclusion were adult patients with newly diagnosed non-squamous NSCLC and synchronous multiple BM, defined as $\geq 3 \mathrm{BM}$ in a period not exceeding 30 days from diagnosis of the primary tumor. We have excluded patients having received any previous systemic or local brain treatment [neurosurgical resection, stereotactic radiosurgery (SRS) or WBRT], those lacking baseline brain MRI availability, exclusively non measurable cerebral disease and those carrying activating mutations of the epidermal growth factor receptor $(E G F R)$ or anaplastic lymphoma kinase (ALK) re-arrangements.

\section{Treatment regimen}

Patients were treated according to the plan defined by the multidisciplinary tumor board, and were classified for the analysis according to the therapeutic approach: upfront chemo alone (group A, n=17) or WBRT plus subsequent chemo (group B, n=11). Group A patients received either intravenous cisplatin $75 \mathrm{mg} / \mathrm{m}^{2}$ or carboplatin [area under the curve (AUC) 5] plus pemetrexed $500 \mathrm{mg} / \mathrm{m}^{2}$ every 3 weeks up to 4-6 cycles followed by maintenance pemetrexed until disease progression or unacceptable toxicity. Group B patients received WBRT with a dose of 30 Gy in 10 fractions daily, followed by systemic chemo as in group A.

Enrolled patients without any neurological deficit received upfront chemo. Whereas patients with the presence of neurologic symptoms received WBRT followed by chemo. Patients with medically, well-controlled, seizures or headache, those with mild paresis of extremities, defined with an Medical Research Council (MRC) score of 4, or pure sensory symptoms without functional impairment were considered as patients without neurological symptoms and were thus allocated in group A to receive monotherapy with upfront systemic chemo.

\section{MRI data}

Brain MRI scans were performed using 1 of 3 MRI systems (Philips Ingenia S 3T with 32-channel head coil, Philips Medical System, Best, The Netherlands). The images obtained and analyzed on $1.5 \mathrm{~T}$ systems were acquired with axial 5 -mm slices with $0.5-\mathrm{mm}$ gap for both gadoliniumenhanced T1-weighted image (T1Gd) and fluid attenuated inversion recovery (FLAIR) sequences, whilst those from
$3 \mathrm{~T}$ system were axial $3-\mathrm{mm}$ reconstructions of a $3 \mathrm{D}$ sagittal acquisition of $0.8 \mathrm{~mm}$ and no gap.

The extension of edema was estimated by calculating the ratio between the maximum diameter in FLAIR and T1Gd sequences $(\mathrm{F} / \mathrm{Gd})$ in the axial plane per each BM depicted at the baseline MRI (see Figure 1). Enrolled patients had an available baseline brain MRI with measurable brain disease according to the response assessment neuro-oncology (RANO) BM criteria, which was used to classify the response to the treatment as progressive disease (PD), stable disease (SD) and partial response (PR) (33).

\section{Statistical analysis}

Descriptive data was presented as mean and standard deviation or median and range for continuous variables, and as observed counts and percentages for categorical variables. Intergroup comparisons were performed using Chi-square, Student's $t$-text and ANOVA depending on the variable's nature. In the cases where the variable did not follow a normal distribution, a Mann Whitney U test was applied. Post-hoc Tukey test was applied when multiple intragroup comparisons were performed. Survival curves were obtained using Kaplan-Meier method and compared by log rank test. Finally, receiver operating characteristic (ROC) analysis was applied to identify the most appropriate cut-off for the F/Gd ratio to predict the BM response. All tests were two-tailed and $\mathrm{P}$ values $<0.05$ were considered significant. Patients missing main variable (baseline MRI) were excluded from the analysis. Statistics analyses were performed using IBM SPSS Statistics Software (Version 25.0; IBM Corp, Armonk, NY, USA).

\section{Results}

\section{Characteristics of the participants}

A total of 54 patients diagnosed with NSCLC BM from 2014 to 2016 were identified, from which 28 (52\%) meet the selection criteria. A total of 26 patients from the core study sample were eventually excluded for the following reasons: previous local or systemic treatment $(\mathrm{n}=7)$, non-available baseline MRI $(n=2)$, exclusively non measurable cerebral disease $(n=4)$ and carrying EGFR or ALK mutations $(\mathrm{n}=13)$. Demographic, clinical, tumor-related data, and OS of the 28 patients finally included are described in Table 1. There were no significant differences between groups for almost all variables. However, the number of patients 

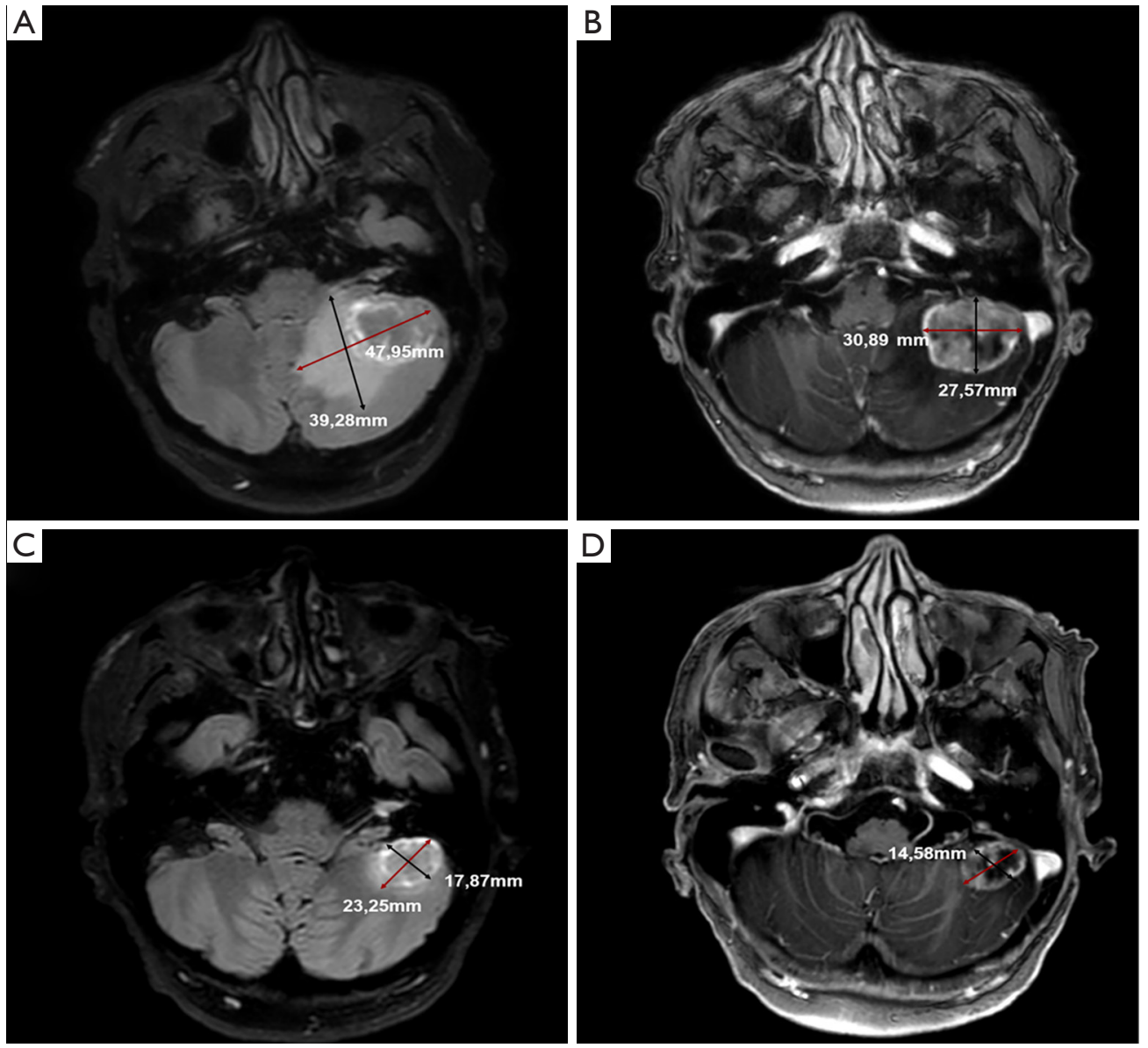

Figure 1 Evaluation of intracranial response before starting chemo treatment (A, FLAIR; B, T1Gd) and after 8 weeks under chemo treatment (C, FLAIR; D, T1Gd). FLAIR, fluid attenuated inversion recovery; T1Gd, gadolinium-enhanced T1-weighted image.

under steroid treatment was clearly superior in the WBRT plus chemo group $(\mathrm{P}=0.007)$. Median progression-free survival (PFS) and OS were comparable between group A and $\mathrm{B}$ (10.6 vs. 8.6 months, $\mathrm{P}=0.449$ for $\mathrm{PFS}$; and 12.9 vs. 10.9 months, $\mathrm{P}=0.317$ for OS, respectively). Among patients allocated in group A, 41\% [7/17] were rescued with WBRT at intracranial progression during their follow-up. Only 3 (11\%) out of 28 evaluable patients were still alive at the moment of data collection.

\section{Neuroimaging findings}

According to the first intracranial response evaluation (3 months after initial treatment), results were distributed as follows: PR 29\% ( $\mathrm{n}=5)$, SD 53\% ( $\mathrm{n}=9)$ and PD 18\% ( $\mathrm{n}=3)$ of patients in group A; and PR 55\% (n=6), SD 27\% ( $n=3)$ and PD $18 \%(n=2)$ of patients in group B. Patients treated with WBRT plus chemo had significantly higher values of F/Gd ratio, compared to those treated with upfront chemo alone ( $2.26 \pm 0.99$ vs. $1.57 \pm 0.62 \mathrm{~mm}$, respectively; $\mathrm{P}=0.008)$.

F/Gd ratio in group A was higher in those who developed intracranial PD $(2.80 \pm 0.32 \mathrm{~mm})$, compared to those who achieved either PR $(1.30 \pm 0.11 \mathrm{~mm})$ or SD $(1.35 \pm 0.09 \mathrm{~mm})$. ANOVA test showed a significant effect on F/Gd ratio between response subgroups at the $\mathrm{P}<0.05$ level $[\mathrm{F}(2,46)=7.749 ; \mathrm{P}=0.001]$. Post-hoc comparisons indicated that the $\mathrm{F} / \mathrm{Gd}$ ratio was significantly higher in $\mathrm{PD}$ subgroup compared to both $\mathrm{SD}$ and $\mathrm{PR}$ subgroups $(\mathrm{P}=0.002$ and $\mathrm{P}=0.001$, respectively).

Conversely, the corresponding F/Gd ratio of group B did not show significant differences according to the BM response status: $\mathrm{PD}(2.25 \pm 0.30), \mathrm{PR}(2.50 \pm 0.21)$ and $\mathrm{SD}$ $(1.40 \pm 0.37)[\mathrm{F}(2,31)=0.061 ; \mathrm{P}=0.941]$.

Additionally, in order to identify the most discriminating 
Table 1 Demographic and clinical data

\begin{tabular}{|c|c|c|c|c|}
\hline Variables & $\begin{array}{l}\text { Whole series } \\
\qquad(\mathrm{N}=28)\end{array}$ & $\begin{array}{l}\text { Group A (chemo) } \\
\qquad(\mathrm{N}=17)\end{array}$ & $\begin{array}{c}\text { Group B (WBRT + chemo) } \\
\qquad(\mathrm{N}=11)\end{array}$ & $P$ value \\
\hline Gender, n (\%) & & & & 0.903 \\
\hline Female & $8[29]$ & $5[29]$ & $3[27]$ & \\
\hline Smoking, pack-year (mean \pm SD) & $35.31 \pm 8.94$ & $32.17 \pm 27.74$ & $40.00 \pm 15.09$ & 0.418 \\
\hline Brain metastases at diagnosis $(\mathrm{n})$, median [range] & 4 [3-5] & 4 [3-5] & 3 [3-5] & 0.697 \\
\hline Patients with extracranial metastases at diagnosis, $\mathrm{n}[\%]$ & 20 [71] & $11[61]$ & $9[80]$ & 0.336 \\
\hline No & $8[29]$ & $8[47]$ & 0 & \\
\hline DS-GPA 1-4, median [range] & $2[1-4]$ & $2[1-4]$ & $2[1-3.5]$ & 0.394 \\
\hline KPS 0-100, median [range] & $90[70-100]$ & $80[70-100]$ & $90[70-100]$ & 0.201 \\
\hline
\end{tabular}

WBRT, whole brain radiotherapy; SD, standard deviation; DS-GPA, diagnosis-specific graded prognostic assessment; KPS, Karnofsky Performance Scale.

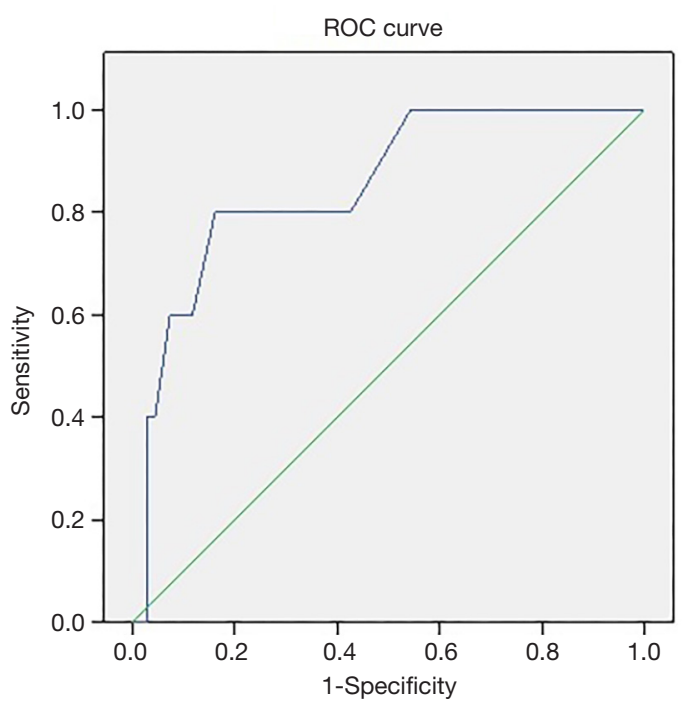

Figure 2 ROC curve plot to predict the $\mathrm{BM}$ response in patients treated with chemo alone according to $\mathrm{F} / \mathrm{G}$ ratio. ROC, receiver operating characteristic; BM, brain metastases; F/Gd, FLAIR/ T1Gd ratio; FLAIR, fluid attenuated inversion recovery; T1Gd, gadolinium-enhanced T1-weighted image. cut-off value for F/Gd ratio, a ROC analysis was performed in group $\mathrm{A}$, showing a 2.1 cut-off value on the $\mathrm{F} / \mathrm{Gd}$ ratio, with a sensitivity of $80 \%$ and a specificity of $83.8 \%$ [AUC $0.851,95 \%$ confidence interval (CI): 0.689-1.014], $\mathrm{P}=0.005$, for benefit as a result of upfront systemic treatment (Figure 2).

\section{Discussion}

Despite the tellingly poor prognosis of cancer patients with multiple $\mathrm{BM}(\geq 3)$, the growing evidence of clinically meaningful intracranial activity of systemic therapies is leading towards a paradigm shift on the optimal sequencing of BM treatment; especially for those patients who are in an oligo or asymptomatic stage and for whom long termsurvivals are expected.

Current knowledge, suggesting that the normal functioning of BBB in BM is disrupted, has opened new avenues to investigate the role of systemic chemo alone or in combination with immunotherapy as an effective and safe frontline treatment (14,25-32). However, the complexity of the 
microenvironment surrounding the tumor cell and tumor cell barrier itself, hinders the chances of an adequate intracranial response. The biomechanical and biochemical signals that exist around the deliberation systems of drugs to the brain is arousing great interest among the scientific community. We have focused on conventional chemo since our explored cohort of patients were treated with this therapy. However, this issue can also be extrapolated to other types of systemic treatments.

To our knowledge this is the first exploratory study that evaluates the extent of peritumoral edema as a potent determinant in predicting resistance to systemic therapy.

To test the hypothesis, we characterized the extension of edema estimated by routine MRI sequences (F/Gd ratio), as a supporting clinical tool to predict the intracranial response in NSCLC patients and synchronous multiple oligosymptomatic BM. Our results showed that cut-off values of $\mathrm{F} / \mathrm{Gd}$ ratio over 2.1 are associated with chemo failure in those oligosymptomatic BM patients, who were treated with chemo alone (group A).

On the contrary, F/Gd ratio failed to be predictive in WBRT plus chemo (group B)-treated patients with symptomatic BM. This effect might be partly explained by the higher proportion of patients in group B treated with corticosteroids. As it is well known, corticosteroids have an anti-inflammatory effect that can contribute to a reduction in the vascular permeability; thus, stabilizing the BBB and consequently making access from chemo to CNS even more challenging. Furthermore, an alternative explanation might have to do with the cytotoxic effect of radiotherapy on the irradiated cells, which it is not dependent on the permeability of the vessels and can also contribute to an increase of the edema extension.

Other small retrospective studies focused on radiosurgery treatment (SRS) alone in brain oligo-metastases from NSCLC have also shown the extent of perilesional edema as a predictive factor of response and brain progression (34,35). In relation to chemo, there is a lack of current studies on radiological predictive markers of intracranial response. However, they have been explored in other systemic therapies. Recently, a retrospective study including patients with de novo BM of EGFR-mutated NSCLC has reported that MRI characteristics, including tumor necrosis, rim enhancement and specific tumor locations, seem to predict treatment response (36). In addition, another recently retrospective study based on melanoma BM patients treated with ICIs, has described MRI radiomic features as a potential biomarker to predict intratumoral heterogeneity and risk of intracranial progression (37).

In order to minimize the heterogeneity of the baseline demographic and clinical characteristics of our study population, we intentionally included patients with the same tumor histology, staging of extra and intracranial disease and treatment naïve. Nonetheless, we have to acknowledge that the study has several limitations. It is an exploratory retrospective study without pre-planned sample size calculation, with the consequent risk of selection and measurement bias. Moreover, we had to apply strict inclusion criteria to obtain a homogenous cohort, decreasing our final sample size. Thus, the unknown power of this sample precludes extract firm conclusions about survival benefits between the two analyzed groups. Finally, the measurement of lesions diameter was also a potential source of bias. In order to reduce it, measurements were performed by two independent properly trained neuroradiologists following RANO BM criteria.

\section{Conclusions}

To summarize, the extension of perilesional edema measured by F/Gd ratio seems a promising tool to identify oligosymptomatic patients with multiple BM in whom upfront monotherapy with chemo should be considered. Therefore, those oligosymptomatic patients might benefit from upfront chemo which will allow WBRT to be postponed, avoiding its detrimental effects on neurocognition and quality of life. Nonetheless, we must handle these preliminary results with caution as prospective and larger sample studies are needed to validate the findings.

\section{Acknowledgments}

Funding: This work was partially supported by a grant from Instituto de Salud Carlos III (ISCIII) (PI18/01253 to MS) and European Regional Development Fund (ERDF). We also thank CERCA programme/Generalitat de Catalunya for Institutional support.

\section{Footnote}

Reporting Checklist: The authors have completed the STROBE reporting checklist. Available at http://dx.doi. org/10.21037/atm-20-6497

Data Sharing Statement: Available at http://dx.doi. org/10.21037/atm-20-6497 
Conflicts of Interest: All authors have completed the ICMJE uniform disclosure form (available at http://dx.doi. org/10.21037/atm-20-6497). Dr. MD reports personal fees from BMS, personal fees from Roche, personal fees from Astrazeneca, outside the submitted work. Dr. NV reports personal fees from Boehringer Ingelheim, personal fees from Roche, personal fees from Lilly, outside the submitted work. Dr. RP reports personal fees from Boehringer Ingelheim, personal fees from Roche, personal fees from Lilly, personal fees from AstraZeneca, personal fees from BMS, personal fees from MSD, outside the submitted work. Dr. EN reports grants and personal fees from Roche, grants and personal fees from Pfizer, personal fees from Lilly, personal fees from Bristol-Myers Squibb, grants and personal fees from Merck Serono, personal fees from Merck Sharp \& Dohme, personal fees from AstraZeneca, personal fees from Amgen, personal fees from Takeda, personal fees from Boehringer Ingelheim, outside the submitted work. The other authors have no conflicts of interest to declare.

Ethical Statement: The authors are accountable for all aspects of the work in ensuring that questions related to the accuracy or integrity of any part of the work are appropriately investigated and resolved. The study was conducted in accordance with the Declaration of Helsinki (as revised in 2013). The study was approved by institutional research committee (PR005/20) and individual consent for this retrospective analysis was waived.

Open Access Statement: This is an Open Access article distributed in accordance with the Creative Commons Attribution-NonCommercial-NoDerivs 4.0 International License (CC BY-NC-ND 4.0), which permits the noncommercial replication and distribution of the article with the strict proviso that no changes or edits are made and the original work is properly cited (including links to both the formal publication through the relevant DOI and the license). See: https://creativecommons.org/licenses/by-nc-nd/4.0/.

\section{References}

1. Gavrilovic IT, Posner JB. Brain metastases: epidemiology and pathophysiology. J Neurooncol 2005;75:5-14.

2. Barnholtz-Sloan JS, Sloan AE, Davis FG, et al. Incidence proportions of brain metastases in patients diagnosed (1973 to 2001) in the Metropolitan Detroit Cancer Surveillance System. J Clin Oncol 2004;22:2865-72.

3. Berghoff AS, Schur S, Füreder LM, et al. Descriptive statistical analysis of a real-life cohort of 2419 patients with brain metastases of solid cancers. ESMO Open 2016;1:e000024.

4. Chamberlain MC, Baik CS, Gadi VK, et al. Systemic therapy of brain metastases: non-small cell lung cancer, breast cancer, and melanoma. Neuro Oncol 2017;19:11-24.

5. Preusser $M$, Winkler F, Valiente $M$, et al. Recent advances in the biology and treatment of brain metastases of nonsmall cell lung cancer: summary of a multidisciplinary roundtable discussion. ESMO Open 2018;3:e000262.

6. Edelman MJ, Belani CP, Socinski MA, et al. Outcomes associated with brain metastases in a three-arm phase III trial of gemcitabine containing regimens versus paclitaxel plus carboplatin for advanced non-small cell lung cancer. J Thorac Oncol 2010;5:110-6.

7. Planchard D, Popat S, Kerr K, et al. Metastatic NonSmall-Cell Lung Cancer: ESMO Clinical Practice Guidelines for diagnosis, treatment and follow-up. Ann Oncol 2018;29:iv192-237.

8. Reck M, Popat S, Reinmuth N, et al. ESMO Guidelines Working Group. Metastatic non-small-cell lung cancer (NSCLC): ESMO Clinical Practice Guidelines for diagnosis, treatment and follow-up. Ann Oncol 2014;25:iii27-39.

9. Chao JH, Phillips R, Nickson JJ. Roentgen-ray therapy of cerebral metastases. Cancer 1954;7:682-9.

10. Horton J, Baxter DH, Olson KB. The management of metastases to the brain by irradiation and corticosteroids. Am J Roentgenol Radium Ther Nucl Med 1971;111:334-6.

11. Mulvenna P, Nankivell M, Barton R, et al. Dexamethasone and supportive care with or without whole brain radiotherapy in treating patients with non-small cell lung cancer with brain metastases unsuitable for resection or stereotactic radiotherapy (QUARTZ): results from a phase 3, non-inferiority, randomised trial. Lancet 2016;388:2004-14.

12. Brown PD, Ahluwalia MS, Khan OH, et al. WholeBrain Radiotherapy for Brain Metastases: Evolution or Revolution? J Clin Oncol 2018;36:483-91.

13. Brown, PD, Jaeckle K, Ballman KV, et al. Effect of Radiosurgery Alone vs Radiosurgery with Whole Brain Radiation Therapy on Cognitive Function in Patients With 1 to 3 Brain Metastases: A Randomized Clinical Trial. JAMA 2016;316:401-9.

14. Gandhi L, Rodríguez-Abreu D, Gadgeel S, et al. Pembrolizumab plus Chemotherapy in Metastatic NonSmall-Cell Lung Cancer. N Engl J Med 2018;378:2078-92.

15. Alsidawi S, Chaudhary R, Karim NA. Frontline Systemic Therapy with Pemetrexed-Platinum in Nonsquamous Non-Small-Cell Lung Cancer With Asymptomatic Brain 
Metastases. Am J Ther 2017;24:e111-20.

16. Barlesi F, Gervais R, Lena H, et al. Pemetrexed and cisplatin as first-line chemotherapy for advanced nonsmall-cell lung cancer (NSCLC) with asymptomatic inoperable brain metastases: a multicenter phase II trial (GFPC 07-01). Ann Oncol 2011;22:2466-70.

17. Robinet G, Thomas P, Breton JL, et al. Results of a phase III study of early versus delayed whole brain radiotherapy with concurrent cisplatin and vinorelbine combination in inoperable brain metastasis of non-small-cell lung cancer: Groupe Francais de Pneumo Cancerologie (GFPC) Protocol 95-1. Ann Oncol 2001;12:59-67.

18. Soria JC, Ohe Y, Vansteenkiste J, et al. Osimertinib in untreated EGFR-mutated advanced non-small-cell lung cancer. N Engl J Med 2018;378:113-25.

19. Gadgeel S, Peters S, Mok T, et al. Alectinib vs crizotinib in treatment-naïve anaplastic lymphoma kinase-positive $(\mathrm{ALK}+)$ non-small-cell lung cancer: CNS efficacy results from the ALEX study. Ann Oncol 2018;29:2214-22.

20. Peters S, Camidge DR, Shaw AT, et al. Alectinib versus crizotinib in untreated ALK positive non-small-cell lung cancer. N Engl J Med 2017;377:829-38.

21. Bachelot T, Romieu G, Campone M, et al. Lapatinib plus capecitabine in patients with previously untreated brain metastases from HER2-positive metastatic breast cancer (LANDSCAPE): a single-group phase 2 study. Lancet Oncol 2013;14:64-71.

22. McArthur GA, Maio M, Arance A, et al. Vemurafenib in metastatic melanoma patients with brain metastases: an open-label, single-arm, phase 2, multicentre study. Ann Oncol 2017;28:634-41.

23. Long GV, Trefzer U, Davies MA, et al. Dabrafenib in patients with Val600Glu or Val600Lys BRAF-mutant melanoma metastatic to the brain (BREAK-MB): a multicentre, openlabel, phase 2 trial. Lancet Oncol 2012;13:1087-95.

24. Davies MA, Saiag P, Robert C, et al. Dabrafenib plus trametinib in patients with BRAF(V600) mutant melanoma brain metastases (COMBI-MB): a multicentre, multicohort, open-label, phase 2 trial. Lancet Oncol 2017;18:863-73.

25. Genentech Inc., Tecentriq (atezolizumab) prescribing information (US FDA). Available online: https://www. gene.com/download/pdf/tecentriq_prescribing.pdf

26. Merck \& Co. Inc., Keytruda (pembrolizumab) prescribing information (US FDA). Available online: https://www.merck. com/product/usa/pi_circulars/k/keytruda/keytruda_pi.pdf

27. Reck M, Rodríguez-Abreu D, Robinson AG, et al. Pembrolizumab versus Chemotherapy for PD-L1Positive Non-Small-Cell Lung Cancer. N Engl J Med
2016;375:1823-33.

28. Paz-Ares L, Luft A, Vicente D, et al. Pembrolizumab plus Chemotherapy for Squamous Non-Small-Cell Lung cancer. N Engl J Med 2018;379:2040-51.

29. Socinski MA, Jotte RM, Cappuzzo F, et al. Atezolizumab for First-Line Treatment of Metastatic Nonsquamous NSCLC. N Engl J Med 2018;378:2288-301.

30. Goldberg SB, Gettinger SN, Mahajan A, et al. Pembrolizumab for patients with melanoma or non-smallcell lung cancer and untreated brain metastases: early analysis of a non-randomised, open-label, phase 2 trial. Lancet Oncol 2016;17:976-83.

31. Besse B, Le Moulec $S$, Mazières J. et al. Bevacizumab in Patients with Nonsquamous Non-Small Cell Lung Cancer and Asymptomatic, Untreated Brain Metastases (BRAIN): A Nonrandomized, Phase II Study. Clin Cancer Res 2015;21:1896-903.

32. Powell SF, Rodríguez-Abreu D, Langer CJ, et al. Pembrolizumab (pembro) plus platinum-based chemotherapy (chemo) in NSCLC with brain metastases: Pooled analysis of KEYNOTE-021, 189, and 407. Ann Oncol 2019;30:v606-7.

33. Lin NU, Lee EQ, Aoyama H, et al. Response assessment criteria for brain metastases: proposal from the RANO group. Lancet Oncol 2015;16:e270-8.

34. Tini $P$, Nardone V, Pastina $P$, et al. Perilesional edema in brain metastasis from non-small cell lung cancer (NSCLC) as predictor of response to radiosurgery (SRS). Neurol Sci 2017;38:975-82.

35. Nardone V, Nanni S, Pastina $P$, et al. Role of perilesional edema and tumor volume in the prognosis of non-small cell lung cancer (NSCLC) undergoing radiosurgery (SRS) for brain metastases. Strahlenther Onkol 2019;195:734-44.

36. Lin CY, Chang CC, Su PL, et al. Brain MRI imaging characteristics predict treatment response and outcome in patients with de novo brain metastasis of EGFR-mutated NSCLC. Medicine (Baltimore) 2019;98:e16766.

37. Bhatia A, Birger M, Veeraraghavan $H$, et al. MRI radiomic features are associated with survival in melanoma brain metastases treated with immune checkpoint inhibitors. Neuro Oncol 2019;21:1578-86.

Cite this article as: Alemany $M$, Domènech $M$, Argyriou AA, Vilariño N, Majós C, Naval-Baudin P, Lucas A, Palmero R, Simó M, Nadal E, Bruna J. Perilesional edema in brain metastases as predictive factor of response to systemic therapy in non-small cell lung cancer patients: a preliminary study. Ann Transl Med 2021;9(8):648. doi: 10.21037/atm-20-6497 